\title{
Síntese de Poli(1,1'-ferrocenilenovinileno) via Acoplamento de McMurry
}

\author{
Camila S. Gonçalves, Yoshio Kawano, Jonas Gruber \\ Instituto de Química, USP
}

\begin{abstract}
Resumo: Com base nas grandes modificações das propriedades de materiais poliméricos resultantes da inclusão de unidades organometálicas, descrevem-se neste trabalho a síntese e a caracterização de um polímero organometálico conjugado com estrutura semelhante à de poli( $p$-fenilenovinileno) (PPV), o qual apresenta grupos 1,1'-ferrocenileno no lugar de 1,4-fenileno em sua cadeia principal. Sintetizado por meio de reação de acoplamento de McMurry de 1,1'-ferrocenodialdeído, o poli(1,1'-ferrocenilenovinileno) (PFV) se apresentou na forma de um sólido de cor laranja, amorfo e insolúvel tanto em solventes polares quanto em apolares. Para caracterização do PFV, empregaram-se os métodos de espectroscopia no infravermelho (FTIR), espectroscopia Raman e análise termogravimétrica (TGA).
\end{abstract}

Palavras-chave: Polímeros condutores, ferroceno, PPV, acoplamento de McMurry.

\section{Synthesis of Poly(1,1'-ferrocenylenevinylene) via McMurry Coupling}

Abstract: Motivated by the influence on the physical and chemical properties of polymeric materials caused by the inclusion of organometallic units, the present work is focused on the synthesis and characterization of an organometallic conjugated polymer with similar structure to poly ( $p$-phenylenevinylene) (PPV), in which the phenylene groups are replaced by ferrocenylene units in the main chain. The polymer, poly(1,1'-ferrocenylenevinylene), (PFV), was prepared via McMurry coupling reaction of 1,1'-ferrocenedialdehyde as an orange and amorphous solid, insoluble both in polar and apolar solvents. The characterization of the polymer was carried out employing the following methods: infrared (FTIR) and Raman spectroscopies and thermogravimetric analysis (TGA).

Keywords: Ferrocene, PPV, conducting polymers, McMurry coupling.

\section{Introdução}

Atualmente, o interesse por polímeros conjugados organometálicos tem aumentado consideravelmente. Isso se deve sobretudo à diversidade de aplicações possíveis: em dispositivos fotovoltaicos ${ }^{[1]}$, diodos emissores de luz ${ }^{[2]}$, ímãs moleculares ${ }^{[3]}$, sensores de gases ${ }^{[4]}$, entre outras. Segundo a literatura, a incorporação de metalocenos a polímeros conjugados altera expressivamente a estrutura eletrônica destes $^{[5]}$.

Com 18 elétrons na camada de valência, o ferroceno é o membro mais estável da série dos metalocenos ${ }^{[6]}$, não sofrendo modificações por exposição à umidade ou ao ar. Pode ser oxidado reversivelmente por via eletroquímica ou pela ação de oxidantes fracos. O ferroceno pode ser considerado um areno rico em elétrons, possuindo reatividade mais acentuada do que a do benzeno. Em reações de Friedel-Crafts, por exemplo, sua reatividade pode alcançar $10^{6}$ vezes a reatividade do benzeno ${ }^{[7]}$. Assim, inúmeros derivados podem ser preparados a partir do ferroceno, de modo a construir estruturas diversas com o emprego deste metaloceno (Figura 1) (8-13]. $^{2}$.

Entre as diversas classes de polímeros conjugados, a família dos poli(arilenovinileno)s (PAVs $)^{[14]}$ (Figura 2), cujo representante mais conhecido é o poli( $p$-fenilenovinileno)
(PPV), tem sido altamente atraente na pesquisa relacionada, inicialmente, com dispositivos eletroluminescentes ${ }^{[15]}$ $\mathrm{e}$, mais recentemente, com células solares ${ }^{[16]}$, transistores magnéticos ${ }^{[17-19]} \mathrm{e}$ narizes eletrônicos ${ }^{[20-22]}$.

A combinação da eletroafinidade dos metais de transição com as propriedades eletrônicas dos polímeros conjugados é uma estratégia atrativa para gerar polímeros híbridos, combinando propriedades físicas, eletrônicas e magnéticas propícias para diversas aplicações. A delocalização eletrônica ao longo da cadeia polimérica pode, aumentar a comunicação entre os centros metálicos, permitindo um ajuste fino das propriedades magnéticas e espectroscópicas para aplicações específicas ${ }^{[23,24]}$. Usualmente, polímeros condutores contendo ferroceno na cadeia principal apresentam interessantes propriedades semicondutoras ${ }^{[25]}$ e magnéticas ${ }^{[26]}$, além de foto-atividade ${ }^{[27]}$, desta forma é possível a obtenção de materiais nanoestruturados e nanoorganizados, com sítios redox ativos e características interessantes para aplicações em dispositivos ópticos, fotônicos e magnéticos ${ }^{[28,29]}$.

Neste artigo, apresentam-se a síntese em uma única etapa, via reação de McMurry, e a caracterização de poli(1,1'-ferrocenilenovinileno) (1) (PFV), polímero análogo ao PPV, que possui grupos 1,1 '-ferrocenileno incorporados à sua cadeia principal (Figura 2). Cabe ressaltar que este polímero já foi descrito uma única vez na literatura ${ }^{[30]}$,

Autor para correspondência: Jonas Gruber, Instituto de Química, Departamento de Química Fundamental, USP, Av. Prof. Lineu Prestes 748, CEP: 05402-970, São Paulo, SP, Brasil.E-mail: jogruber@iq.usp.br 


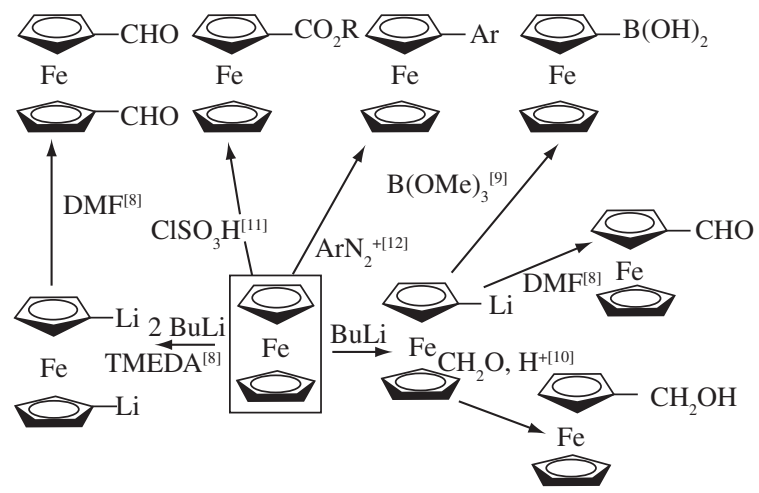

Figura 1. Alguns exemplos de reações envolvendo ferroceno.

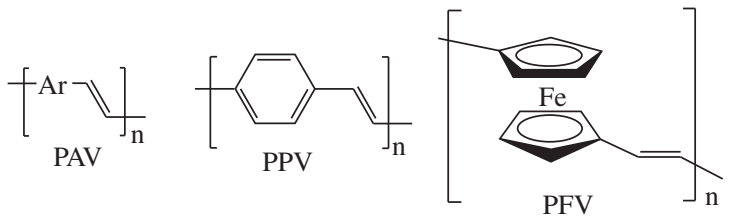

Figura 2. Fórmulas estruturais de PAV, PPV, PFV.

tendo sido obtido via polimerização por metátese e abertura de anel (ROMP), que envolve duas etapas sintéticas e longo tempo de reação.

\section{Experimental}

\section{Instrumentos utilizados e condições de obtenção}

Os espectros no infravermelho foram obtidos por meio de espectrômetro da Perkin-Elmer modelo 1750 com transformada de Fourier utilizando a amostra na forma de pastilha de $\mathrm{KBr}$.

O espectro de FT-Raman foi feito em um espectrômetro Bruker modelo FTS100/S, equipado com um laser contínuo Nd-YAG (1064 nm e potência de $100 \mathrm{~mW}$ ) e um detector de Ge resfriado com nitrogênio líquido. A resolução foi de $4 \mathrm{~cm}^{-1}$ e 256 co-adições.

A termogravimetria foi realizada sob atmosfera dinâmica de ar, com razão de aquecimento de $20{ }^{\circ} \mathrm{C} \mathrm{min}{ }^{-1}$, resolução 3 , massa de amostra entre 3,00 e 6,00 mg. Utilizou-se uma termobalança Hi-Res ${ }^{\text {TM }}$ TGA 2950 da TA Instruments.

\section{Preparação de precursores}

1,1'-Ferrocenodialdeído (1) foi obtido a partir do ferroceno por meio de geração do derivado dilitiado e subseqüente reação com $N, N$-dimetilformamida (DMF), de acordo com procedimento descrito na literatura ${ }^{[31,32]}$.

\section{Síntese de PFV (1)}

Em um balão de três bocas de $50 \mathrm{~mL}$, equipado com condensador de refluxo, adicionou-se, a uma suspensão de pó de zinco $(1,21 \mathrm{~g}, 18,5 \mathrm{mmol})$ em $20 \mathrm{~mL}$ de tetrahidrofurano (THF), tetracloreto de titânio $(0,65 \mathrm{~mL}, 5,9 \mathrm{mmol})$ a $-10{ }^{\circ} \mathrm{C}$ sob atmosfera de $\mathrm{N}_{2}$ seco. Após a formação de uma sus- pensão esverdeada, acrescentou-se 0,612 g (2,53 mmol) de 1,1'-ferrocenodialdeído (1) solubilizado em $14 \mathrm{~mL}$ de THF.

A mistura foi aquecida a refluxo por 9 horas, resfriada e vertida sobre $40 \mathrm{~mL}$ de uma solução aquosa de carbonato de potássio a $10 \%$. A mistura resultante foi agitada por 30 minutos e o sólido formado na forma de um filme laranja foi separado, lavado com água e secado, rendendo $0,185 \mathrm{~g}(0,995 \mathrm{mmol} ; 39 \%)$ de PFV. FTIR $\left(\mathrm{cm}^{-1}, \mathrm{KBr}\right)$ : $3085\left(v_{\mathrm{C}-\mathrm{H}}\right.$ anel ciclopentadienílico - $\left.\mathrm{cp}\right), 1701\left(\mathrm{v}_{\mathrm{C}=\mathrm{O}}\right)$ e 1629 $\left(v_{\mathrm{C}=\mathrm{C}}\right), 1443$ e $1379\left(\mathrm{v}_{\mathrm{C}-\mathrm{C}} \mathrm{cp}\right), 1187\left(\mathrm{v}_{\mathrm{C}-\mathrm{C}} \mathrm{cp}\right), 1030\left(\delta_{\mathrm{C}-\mathrm{H}} \mathrm{cp}\right)$, $956\left(\delta_{\mathrm{H}-\mathrm{C}=\mathrm{C}-\mathrm{H}}-\operatorname{trans}\right), 859\left(\delta_{\mathrm{H}-\mathrm{C}=\mathrm{C}-\mathrm{H}}-\right.$ cis $), 805\left(\delta_{\mathrm{C}-\mathrm{H}} \mathrm{cp}\right)$. Raman $\left(\mathrm{cm}^{-1}\right): 1309\left(\delta_{\mathrm{H}-\mathrm{C}=\mathrm{C}-\mathrm{H}}-\right.$ trans $), 1235\left(\delta_{\mathrm{H}-\mathrm{C}=\mathrm{C}-\mathrm{H}}-\right.$ cis $)$. TGA: $303{ }^{\circ} \mathrm{C}(\Delta \mathrm{m}=-3 \%) ; 336{ }^{\circ} \mathrm{C}(\Delta \mathrm{m}=-8 \%) ; 408{ }^{\circ} \mathrm{C}$ $(\Delta \mathrm{m}=-19 \%) ; 539{ }^{\circ} \mathrm{C}(\Delta \mathrm{m}=-57 \%) ; 787{ }^{\circ} \mathrm{C}(\Delta \mathrm{m}=-62 \%) . \mathrm{O}$ polímero se mostrou insolúvel em diversos solventes como água, acetona, DMSO, acetonitrila, DMF, álcool etílico, acetato de etila, hexano, éter de petróleo, diclorometano e éter etílico, mesmo sob aquecimento.

\section{Resultados e Discussão}

Diversas rotas sintéticas para o PFV foram testadas, conforme ilustrado na Figura 3. As rotas A, B, C e D partiram de ferroceno (1) ou de 1,1'-dimetilferroceno (2), ambos de procedência comercial, a fim de preparar precursores tetrahalogenados (3 ou 4) cujas reduções catódicas levariam à formação de PFV, a exemplo do que ocorre com inúmeros derivados aromáticos tetrahalogenados convertidos eletroquimicamente aos respectivos poli( $p$-fenileno vinileno)s, $\mathrm{PPVS}^{[33,34]}$. A instabilidade dos 1,1'-bis(halometil)ferrocenos (5 e 6) e 1,1'-bis(dibromometil)ferroceno (3), entretanto, resultante da rápida hidrólise dos grupos halometila ${ }^{[35]}$, ou da fácil oxidação das unidades ferrocênicas ${ }^{[31]}$, não permitiu obter PFV por essas rotas.

A rota $\mathrm{E}$ foi a única a permitir a preparação de PFV. Assim, o tratamento de ferroceno (1) com $n$-butil-lítio, seguido de DMF, conduziu a 1,1'-ferrocenodialdeído (7), cujo acoplamento de McMurry ${ }^{[36]}$, induzido por titânio, forneceu PFV em rendimento de $36 \%$.

Com base no espectro infravermelho do PFV foi possível verificar: a) a estereoquímica dos grupos vinilena por meio da presença de bandas de deformação angular fora do plano $=\mathrm{C}-\mathrm{H}$ em 956 e em $859 \mathrm{~cm}^{-1}$, que sugerem configuração trans e cis, respectivamente; b) a presença de grupos terminais $\mathrm{C}(=\mathrm{O}) \mathrm{H}$, evidenciada por uma banda fraca em $1701 \mathrm{~cm}^{-1}$; e c) a presença dos anéis de ferroceno por meio de bandas de estiramento da ligação $\mathrm{C}-\mathrm{H}$ em $3085 \mathrm{~cm}^{-1}$, entre outras $^{[37]}$.

O espectro Raman permitiu confirmar a presença de duplas ligações com configuração trans e cis por meio de bandas características em 1309 e $1235 \mathrm{~cm}^{-1}$, respectivamente ${ }^{[38]}$.

A estabilidade térmica em atmosfera de ar pôde ser avaliada por termogravimetria, que revelou um início de perda de massa em $303{ }^{\circ} \mathrm{C}$. A partir desta temperatura, a decomposição foi lenta, intensificando-se depois de $408{ }^{\circ} \mathrm{C}$, sen- 


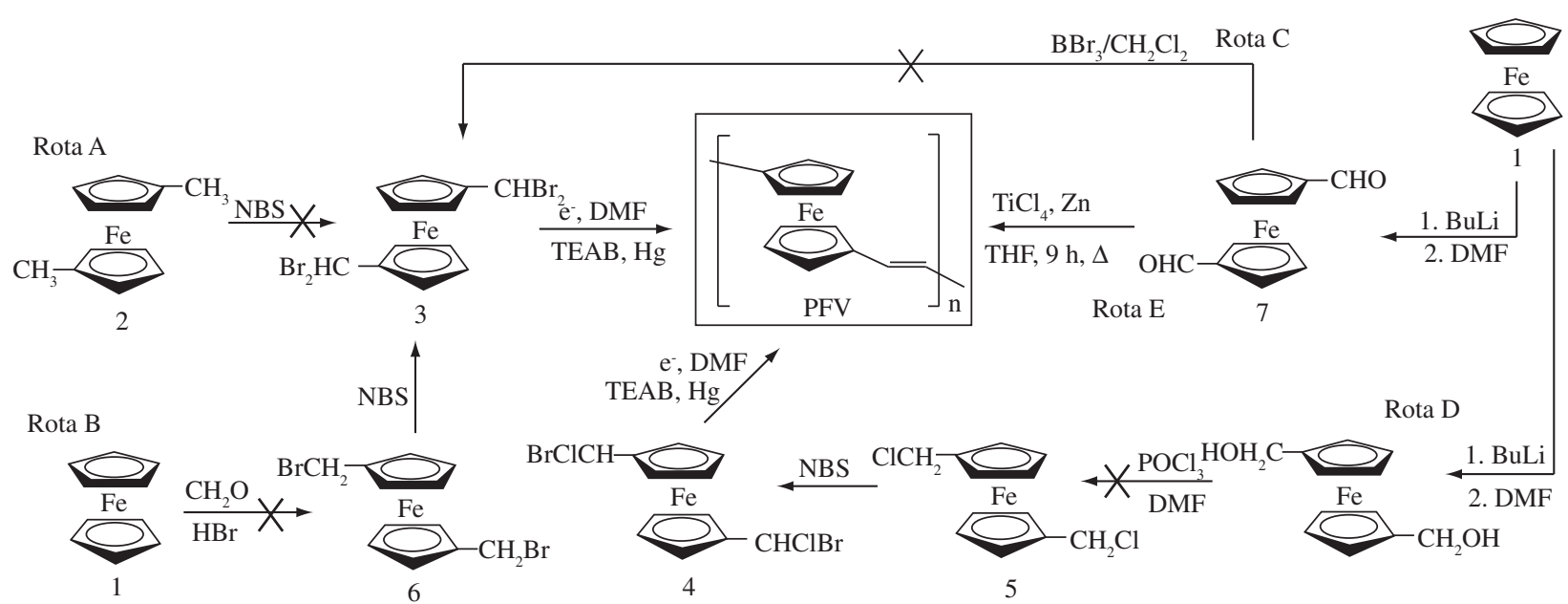

Figura 3. Rotas seguidas para a síntese de PFV.

do $47 \%$ da massa eliminada em $539^{\circ} \mathrm{C}$. Foi observada uma massa residual de $11,7 \%$ em $730{ }^{\circ} \mathrm{C}$, que pode ser atribuída à formação de óxido férrico $(9 \%)$ e de algum outro resíduo não volátil $(2,7 \%)$.

Tendo em vista a insolubilidade do PFV, as aplicações que envolvem a necessidade de formação de filmes finos (sensores, dispositivos opto-eletrônicos, etc.) podem ser conseguidas via deposição do polímero por sublimação a pressão reduzida ${ }^{[39]}$. Outras possibilidades seriam a preparação do PFV substituído por cadeias laterais longas ${ }^{[25]}$, ou a copolimerização entre o monômero contendo ferroceno, (i.e. 1,1'-ferrocenodialdeído) e monômeros de outra natureza, mas que contenham cadeias longas (p. ex. 2,5-di- $n$-octiloxibenzeno1,4-dialdeído) ${ }^{[40]}$.

\section{Conclusões}

Poli(1,1'-ferrocenilenovinileno), PFV, um polímero organometálico conjugado, foi sintetizado com rendimento de 36\% por meio de reação de acoplamento de McMurry. Análises espectroscópicas no infravermelho e Raman revelaram que sua estrutura apresenta duplas vinilênicas de configuração cis e trans. A estabilidade térmica do polímero foi avaliada por termogravimetria (temperatura de início da decomposição $\left.=303{ }^{\circ} \mathrm{C}\right)$.

\section{Agradecimentos}

À FAPESP e ao CNPq pelo suporte financeiro. À Simone Zaccarias pela revisão do manuscrito.

\section{Referências Bibliográficas}

1. Cyr, P. W.; Klem, E. J. D.; Sargent, E. H. \& Manners, I. - Chem. Mater., 17, p.5770 (2005).

2. (a) Koga, Y.; Ueno, K. \& Matsubara, K. - J. Polym. Sci., Part A: Polym. Chem., 44, p.4204 (2006). (b) Dennany,
L.; Hogan, C. F.; Keyes, T. E. \& Forster, J. - Anal. Chem., 78, p.1412 (2006).

3. Clendenning, S. B.; Fournier-Bidoz, S.; Pietrangelo, A.; Yang, G.; Han, S.; Brodersen, P. M.; Yip, C. M.; Lu, Z.-H.; Ozin, G. A. \& Manners, I. - J. Mater. Chem., 14, p.1686 (2004).

4. Penza, M.; Cassano, G.; Sergi, A.; Lo Sterzo, C. \& Russo, V. - Sens. Actuators B, B81, p.88 (2001).

5. (a) Caliendo, C.; Verona, E.; D’Amico, A.; Furlani, A.; Infante, G. \& Russo, M. V. - Sens. Actuators B, 2425, p.670 (1995). (b) Matsuura, Y. \& Matsukawa, K. - Chem. Phys. Lett., 428, p.321 (2006).

6. Wilkenson, G.; Rosenblum, M.; Whiting, M. C. \& Woodward, R. B. - J. Am. Chem. Soc., 74, p.2123 (1952).

7. Bochmann, M. - "Organometallics 2: Complexes with transition metal - carbon $\pi$-bonds (Oxford Chemistry Primers 45)", Oxford University Press, Oxford (1996).

8. Fröhlich, R. F. G.; Zabelinskaja-Mackova, A. A.; Fechter, M. H. \& H. Griengl - Tetrahedron: Asymmetry, 14, p.355 (2003).

9. Carroll, M. A.; Widdowson, D. A. \& Williams, D. J. Synlett, p.1025 (1994).

10. Knapp, R. \& Rehahn, M. - J. Organomet. Chem., 452, p.235 (1993).

11. Carlström, A. \& Frejd, T. - J. Org. Chem., 55, p.4175 (1990).

12. Reeves, P. C. - Org. Synth., Coll. 6, p.625 (1988).

13. Rosenblum, M.; Howells, W.G.; Banerjee, A. K. \& Bennett, C. - J. Am. Chem. Soc., 84, p.2726 (1962).

14. Gruber, J.; Li, R. W. C. \& Hümmelgen, I. A. - "Synthesis, Properties and Applications of Poly(p-Phenylene Vinylenes)s", in: Handbook of Advanced Electronic and Photonic Materials and Devices, cap. 4, vol. 8, Nalwa, H. S. (ed.), Academic Press, London, (2001). 
15. Akcelrud, L. - Prog. Polym. Sci., 28, p.875 (2003).

16. Gruber, J.; Li, R. W C.; Aguiar, L. H. J. M. C.; Garcia, T. L.; de Oliveira, H. P. M.; Atvars, T. D. Z. \& Nogueira, A. F. - Synth. Met., 156, p.104 (2006).

17. Meruvia, M. S.; Benvenho, A. R. V.; Hümmelgen, I. A.; Li, R. W. C.; Aguiar, L. H. J. M. C. \& Gruber, J. - Solid State Commun., 139, p.27 (2006).

18. Meruvia, M. S.; Benvenho, A. R. V.; Hümmelgen, I. A.; Gomez, J. A.; Graeff, C. F. O.; Li, R. W. C.; Aguiar, L. H. J. M. C. \& Gruber, J. - Phys. Stat. Sol. A, 202, p.R158 (2005).

19. Meruvia, M. S.; Freire, J. A.; Hümmelgen, I. A.; Gruber, J. \& Graeff, C. F. O. - Org. Electr., 8, p. 695 (2007).

20. Gruber, J.; Yoshikawa, E. K. C.; Bao, Y. \& Geise, H. J. e-Polymers, 14, p.1-9 (2004).

21. Rosa, R. M.; Szulc, R. L.; Li , R. W. C. \& Gruber, J. Macromol. Symp., 229, p.138 (2005).

22. Péres, L. O. \& Gruber, J. - Mater. Sci. Eng. C, 27, p.67 (2007).

23. Hirao, T.; Kurashina, M.; Aramaki, K. \& Nishihara, H. J. Chem. Soc., Dalton Trans., 14, p.2929 (1996).

24. Southard, G. E. \& Curtis, M. D. - Organometallics, 20, p.508 (2001).

25. Itoh, T.; Saitoh, H. \& Iwatsuki, S. - J. Polym. Sci., Part A: Polym. Chem., 33, p.1589 (1995).

26. Takahashi, S.; Takai, Y.; Morimoto, H. \& Sonogashira, K. - J. Chem. Soc., Chem. Commun., p.3 (1984).

27. Gooding, R.; Lillya, C. P. \& Chien, C. W - J. Chem. Soc., Chem. Commun., p.151 (1983).

28. Chen, T.; Wang, L.; Jiang, G.; Wang, J.; Wang, X.; Zhou, J. \& Wang, W. - Eur. Pol. J., 42, p.687 (2006).
29. Wang, X.; Arsenault, A.; Ozin, G. A.; Winnik, M. A. \& Manners, I. - J. Am. Chem. Soc., 125, p.12686 (2003).

30. Buretea, M. A. \& Tilley, T. D. - Organometallics, 16, p.1507 (1997).

31. Fröhlich, R. F. G.; Zabelinskaja-Mackova, A. A.; Fechter, M. H. \& Griengl, H. - Tetrahedron: Asymmetry, 14, p.355 (2003).

32. Carroll, M. A.; Widdowson, D. A. \& Williams, D. J. Synlett, p.1025 (1994).

33. Utley, J. H. P. \& Gruber, J. - J. Mater. Chem., 12, p.1613 (2002).

34. Gruber, J.; Li, R. W. C.; Aguiar, L. H. J. M. C.; Benvenho, A. R. V.; Lessmann, R. \& Hümmelgen, I. A. - J. Mater. Chem., 15, p.517 (2005).

35. Lansinger, J. M. \& Ronald, R. C. - Synth. Commun., 9, p.341 (1979).

36. McMurry, J.E. \& Flemming, M.P. - J. Am. Chem. Soc., 96, p.4708 (1974).

37. Fritz, H. P. - Chem. Ber. 92, p.780 (1959).

38. Liao, L.; Pang, Y.; Ding, L. \& Karasz, F. E. - Macromolecules, 34, p.6756 (2001).

39. Plank, R. V.; Wei, Y.; DiNardo, N. J. \& Vohs, J. M. Chem. Phys. Lett., 263, p.33 (1996).

40. Gonçalves, C. S.; Serbena, J. P.; Hümmelgen, I. A. \& Gruber, J. - Macromol. Symp., 245-246, p.22 (2006).

Enviado: 01/04/07

Reenviado: 10/08/07

Aceito: 20/08/07 